\title{
Bur open Suicide following the death of a sibling: a nationwide follow-up study from Sweden
}

\author{
Mikael Rostila, ${ }^{1}$ Jan Saarela, ${ }^{2}$ Ichiro Kawachi ${ }^{3}$
}

To cite: Rostila M, Saarela J, Kawachi I. Suicide following the death of a sibling: a nationwide follow-up study from Sweden. BMJ Open 2013;3:e002618.

doi:10.1136/bmjopen-2013002618

- Prepublication history this paper are available online. To view these files please visit the journal online (http://dx.doi.org/10.1136/ bmjopen-2013-002618).

Received 21 January 2013 Revised 28 February 2013 Accepted 21 March 2013

This final article is available for use under the terms of the Creative Commons Attribution Non-Commercial 2.0 Licence; see http://bmjopen.bmj.com

${ }^{1}$ Centre for Health Equity Studies (CHESS), Stockholm University/Karolinska Institutet, Stockholm, Sweden

${ }^{2}$ Åbo Akademi University and University of Helsinki, Vasa, Finland

${ }^{3}$ Harvard School of Public Health, Boston,

Massachusetts, USA

Correspondence to Dr Mikael Rostila; mikael.rostila@chess.su.se

\section{ABSTRACT}

Objectives: The death of a sibling can trigger grief and depression. Sibling deaths from external causes may be particularly detrimental, since they are often sudden. We aimed to examine the association between the death of an adult sibling from external causes and the risk of suicide among surviving siblings up to 18 years after bereavement. We adjusted for intrafamily correlation in death risks, which might occur because of shared genetics and shared early-life experiences of siblings in the same family.

Design: A follow-up study between 1981 and 2002 based on the total population.

Setting: Sweden.

Participants: Swedes aged 25-64 years ( $n=1748$ 069).

Primary and secondary outcome measures:

Suicide from the Swedish cause of death register.

Results: An increased risk of mortality from suicide was found among persons who had experienced the death of a sibling. In women, the suicide risk was 1.55 times that of non-bereaved persons $(95 \% \mathrm{Cl} 0.99$ to $2.44)$, and in men it was 1.28 times higher $(95 \% \mathrm{Cl}$ 0.93 to 1.77 ). If one sibling committed suicide, the risk of the remaining sibling also committing suicide was $3.19(95 \% \mathrm{Cl} 1.23$ to 8.25$)$ among women and $2.44(95 \% \mathrm{Cl} 1.34$ to 4.45$)$ among men. Associations with other main causes of death-such as external other than suicide, cardiovascular diseases or cancer-were generally much smaller and statistically not significant in either sex. We found no clear support for a specific time pattern according to time since a sibling's death.

Conclusions: Our study provided evidence for suicide risk associated with the death of a sibling at adult age, revealing that bereaved persons' risk of suicide is higher when siblings die from suicide, even when adjusting for intrafamily correlation in death risks.

\section{INTRODUCTION}

Previous studies have established that the loss of a family member leads to an increased risk of death and disease among bereaved family members such as spouses, parents and children. ${ }^{1-5}$ Bereavement also contributes to

\section{ARTICLE SUMMARY}

Article focus

- The loss of a family member has been linked to increased vulnerability of psychiatric disorders that may be highly associated with suicidal behaviour.

- The impact of losing an adult sibling on suicide risk of remaining siblings has been largely overlooked.

- Unexpected causes of deaths may precipitate a more severe reaction compared with deaths following chronic illness in which family members have more time to adjust and cope with the loss.

Key messages

- This study provided the first large-scale evidence for suicide associated with sibling death at adult age.

- Even when adjusting for intrafamily correlation in death risks, bereaved persons' suicide risk is raised when their siblings had died from suicide.

Strengths and limitations of this study

- The use of total population register data on sibling groups provides a large sample size, Iongitudinal follow-up, reliable information on deaths from suicide and other included variables.

- The mechanisms linking the death of a sibling and completed suicide among the bereaved person could not be investigated. For instance, the quality of the relationship with the deceased sibling could decide the severity of bereavement and thereby contribute to suicide risk among the remaining siblings.

a higher risk of suicide in bereaved family members. ${ }^{6-10}$ However, the impact of grief following the loss of an adult sibling has been largely overlooked. To the extent that siblings are also beloved, provide companionship and support, one would expect that death of an adult sibling - as much as the death of other family members (eg, spouse, parents, children) - would be associated with an increased risk of suicide. In fact, the death of a sibling often represents the loss of 
the longest and most intimate relationships of a person's lifetime. ${ }^{11}$ Some studies even suggest that the death of a sibling is more disruptive and involves a more severe grief process than other familial deaths. ${ }^{5} 12$

Interpersonal loss and disruption of attachments from any cause could elevate the risk for suicide, ${ }^{13-15}$ both directly as a proximal precipitant for suicide as well as through the onset or exacerbation of psychiatric illness in survivors. ${ }^{13}$ Consequently, the loss of a family member has been linked to increased vulnerability of psychiatric disorders that may be highly associated with suicidal behaviour, such as major depression and anxiety disorders in adults. ${ }^{13} 1617$ However, the grief reaction associated with sibling loss could be expected to vary according to the cause of death. Thus, sudden or unexpected causes of deaths (eg, suicides, accidents and homicides) may precipitate a more severe reaction compared with deaths following chronic illness (eg, cancer) in which the family members have more time to adjust and cope with the loss. It has, for instance, been found that relatives exposed to deaths from external causes are at a greater risk of developing complicated grief and post-traumatic stress symptoms ${ }^{18-22}$ because such deaths may involve higher immediate stress levels, stronger feelings of grief, greater difficulty in accepting the death and fewer available coping strategies to deal with the loss. ${ }^{23}{ }^{24}$ Accordingly, studies suggest that the loss of a spouse or a parent due to external causes increase the risk of suicide among the bereaved family members. ${ }^{2}$ Furthermore, the time since the death of a family member could be crucial for the risk of suicide after bereavement. For instance, the risk of suicide increases during the first year after the loss of a spouse and decreases thereafter until it is similar to the overall rate. ${ }^{8}$ Men appear to be most at risk during the first few months after bereavement, and for women the risk is distributed more evenly over a longer period. ${ }^{25}$ Whether a similar pattern can be discerned on the suicide risk of bereaved siblings is unknown.

Our aim was to conduct a large-scale longitudinal study on mortality from suicide following the loss of an adult sibling, using intergenerational linked data from the nationwide Swedish registers. We postulated that the association between sibling's death and suicide will depend on the main cause of the sibling's death (suicide or other cause), the gender of the remaining sibling and potentially also the time since the sibling's death. Death risks of siblings and suicide risks, in particular, are necessarily inter-related because of shared genetics and shared early life experiences. We therefore adjusted for intrafamily correlation in death risks using Cox models with random effects.

\section{METHOD}

This study is based on multiple-linked data of the national Swedish routine registers including the total population register (TPR), National Population and
Housing Censuses, the Longitudinal Data Base on Education, Income and Employment (LOUISE) and the Cause of Death registers. The data are maintained at the Centre for Health Equity Studies in Stockholm and was approved by the Regional Ethical Review Board of Karolinska Institutet in 11 November 2002 (decision no. 02-481) and the Central Ethical Review Board 2012-09-13 (application no. 2012/1260-31). These decisions approve that the data can be used for several purposes. All data used for this study were also anonymous and researchers did not have access to any personal information that could identify the study participants (eg, personal identity number, home address, etc). Consequently, it was not possible to trace specific individuals included in the data material.

In the study, all persons born in Sweden during the period 1932-1962 and alive at the end of 1980 were linked to the mother, provided that she was born in Sweden and alive at the same time. Hence, the sibling groups were identified through the mother, and singletons were excluded from analysis. To get a reasonable age balance and in order to use adequate control variables, we restricted the sample to people aged 25-64 years. These study persons were observed from 1981 until 2002.

We included individual-level information about basic sociodemographic variables (age, socioeconomic status, marital status, number of children, number of siblings, region of residence and calendar year) to proxy social and regional differences in suicide, and the month and specific cause of death for all persons who died during the study period. Socioeconomic status distinguished blue-collar workers, white-collar workers, self-employed and people outside the labour market. Marital status consisted of the categories married, previously married and never married. Number of children and number of siblings were treated as categorical variables. Region of residence refers to each person's county of residence and consisted of 26 different categories. Bereaved persons were slightly older than non-bereaved persons, somewhat more of them had a lower socioeconomic position, and they had more siblings, which is expected considering that the likelihood of observing a sibling's death must be higher in larger sibling groups (not shown). We accounted for the distributional differences between bereaved and non-bereaved persons by using the control variables, which generally provided good statistical fit. All covariates except age and calendar year were measured at the end of 1980, which antedated any sibling death. We distinguished sibling deaths from suicide (International Classification of Diseases (ICD) 8 and ICD9 codes E950-E959, and ICD10 codes X60-X84), external causes other than suicide (ICD8 codes E807-E949 and E960-E999, ICD9 codes E800-E949 and E960-E999, and ICD10 codes V01-X59 and X85-Y98), cardiovascular diseases (ICD8 codes 410-438 and 795, ICD9 codes 410-438 and 798, and ICD10 codes I21-I52 and I60-I69), cancer (ICD8 and ICD 9 codes 140-239, and ICD10 codes C00-D48) and all other causes (all other codes). 
Table 1 Descriptive statistics by sex of the index persons

\begin{tabular}{|c|c|c|}
\hline & Men & Women \\
\hline Number of sibling deaths & 78297 & 76910 \\
\hline$\%$ from suicide & 8.7 & 8.9 \\
\hline $\begin{array}{l}\% \text { from external other than } \\
\text { suicide }\end{array}$ & 10.8 & 10.8 \\
\hline$\%$ from cardiovascular diseases & 22.0 & 22.1 \\
\hline$\%$ from cancer & 37.3 & 37.2 \\
\hline \multicolumn{3}{|l|}{ Number of deaths from suicide } \\
\hline In bereaved persons & 242 & 125 \\
\hline In non-bereaved persons & 4376 & 1761 \\
\hline \multicolumn{3}{|l|}{ Number of person-years in } \\
\hline Bereaved persons & 620584 & 611382 \\
\hline Non-bereaved persons & 17893475 & 17308392 \\
\hline \multicolumn{3}{|l|}{ Death rate $(\times 1000)$} \\
\hline In bereaved persons & 0.39 & 0.20 \\
\hline In non-bereaved persons & 0.25 & 0.10 \\
\hline $\begin{array}{l}\text { Number of (ever) bereaved } \\
\text { persons }\end{array}$ & 79017 & 77614 \\
\hline Number of non-bereaved persons & 812186 & 779252 \\
\hline
\end{tabular}

All people who experienced a sibling's death during the study period were included, whereas those who did not experience a sibling's death comprised a $10 \%$ random sample. In the statistical analyses, people from each group were weighted according to their sampling proportion. Normalised weights were used to correct for inflated t-statistics. The death of the sibling was a timevarying feature, which means that when a sibling died, the surviving sibling changed status from being a nonbereaved to being a bereaved person. Using Cox regressions with random effects, ${ }^{26}$ we estimated the suicide risks of bereaved persons to non-bereaved persons. These models adjusted for intrafamily correlations in suicide risks caused by shared genetics and shared family life experiences of siblings from the same family.

\section{RESULTS}

In total, 79017 men and 77614 women experienced a sibling's death, and, respectively, 242 and 125 of them subsequently died from suicide (table 1). The corresponding numbers in non-bereaved persons were 4376 suicide deaths among 812186 women and 1761 suicide deaths among 779252 men. The death rate in bereaved persons was consequently approximately twice that of non-bereaved persons $(0.39 / 0.25$ for men and 0.20/0.10 for women). About $9 \%$ of all sibling deaths were from suicide.

Suicide was more than twice as common in men (4618 deaths in total) as in women (1886 in total), but the association between sibling loss and suicide was greater for women than in men. In bereaved women, the suicide risk was 1.55 times that of non-bereaved women (95\% CI 0.99 to 2.22). In bereaved men, the suicide risk was 1.28 times that of non-bereaved men (0.93 to 1.77; table 2). In particular, those who had experienced a sibling's death from suicide had an own high suicide risk: 3.19 times higher among women (1.23 to 8.25) and 2.44 higher among men (1.34 to 4.45). Even if the sibling died of another external cause than suicide, there was an elevated suicide risk for the surviving sibling: 1.38 times higher among women and 1.21 times among men. The estimates were statistically not significant, however. The same caveat applies to more detailed categorisations of the cause of death. There seemed to be a link also to sibling deaths from external causes other than suicide. Men with this experience had an own risk of suicide that was 1.41 times that of non-bereaved men, whereas the corresponding relative risk in women was even higher, or 1.71. Sibling deaths from physical disease, such as cardiovascular disease or cancer, displayed even weaker associations. CIs were although far too wide to permit any detailed insights.

We also found some tendency towards a pattern where the variation across age groups in the bereavement effect on suicide mortality was found to be smaller for women than for men (results not shown). This might reflect that younger women were more vulnerable to the death of a sibling than young men were. Women also appeared to exhibit somewhat stronger associations in the long term than men (figure 1). In men, the suicide

Table 2 Association between sibling's death from different main causes and suicide in index persons

\begin{tabular}{llr}
\hline & Men & Women \\
\hline Cause of sibling's death & & \\
All causes & $1.28(0.93$ to 1.77$)$ & $1.55(0.99$ to 2.44$)$ \\
Suicide & $2.44(1.34$ to 4.45$)$ & $3.19(1.23$ to 8.25$)$ \\
Not suicide & $1.21(0.88$ to 1.71$)$ & $1.38(0.84$ to 2.26$)$ \\
External other than suicide & $1.41(0.69$ to 2.86$)$ & $1.71(0.63$ to 4.68$)$ \\
Cardiovascular diseases & $1.27(0.66$ to 2.44$)$ & $1.34(0.49$ to 3.69$)$ \\
Cancer & $1.20(0.70$ to 2.05$)$ & $1.02(0.41$ to 2.52$)$ \\
All other causes & $1.08(0.54$ to 2.16$)$ & $1.68(0.72$ to 3.96$)$ \\
\hline
\end{tabular}

Numbers are mortality risk ratios (with $95 \% \mathrm{Cls}$ ), that is, the ratio of the death risk of bereaved persons and non-bereaved persons, adjusted for the effects of all control variables.

Control variables included in the estimations are age, calendar year, socioeconomic status, marital status, number of children, number of siblings and region of residence.

Results are from Cox models with random effects. 
Figure 1 Suicide risk after sibling's death as compared with non-bereaved persons (with 95\% Cls). Results are from Cox models with random effects.
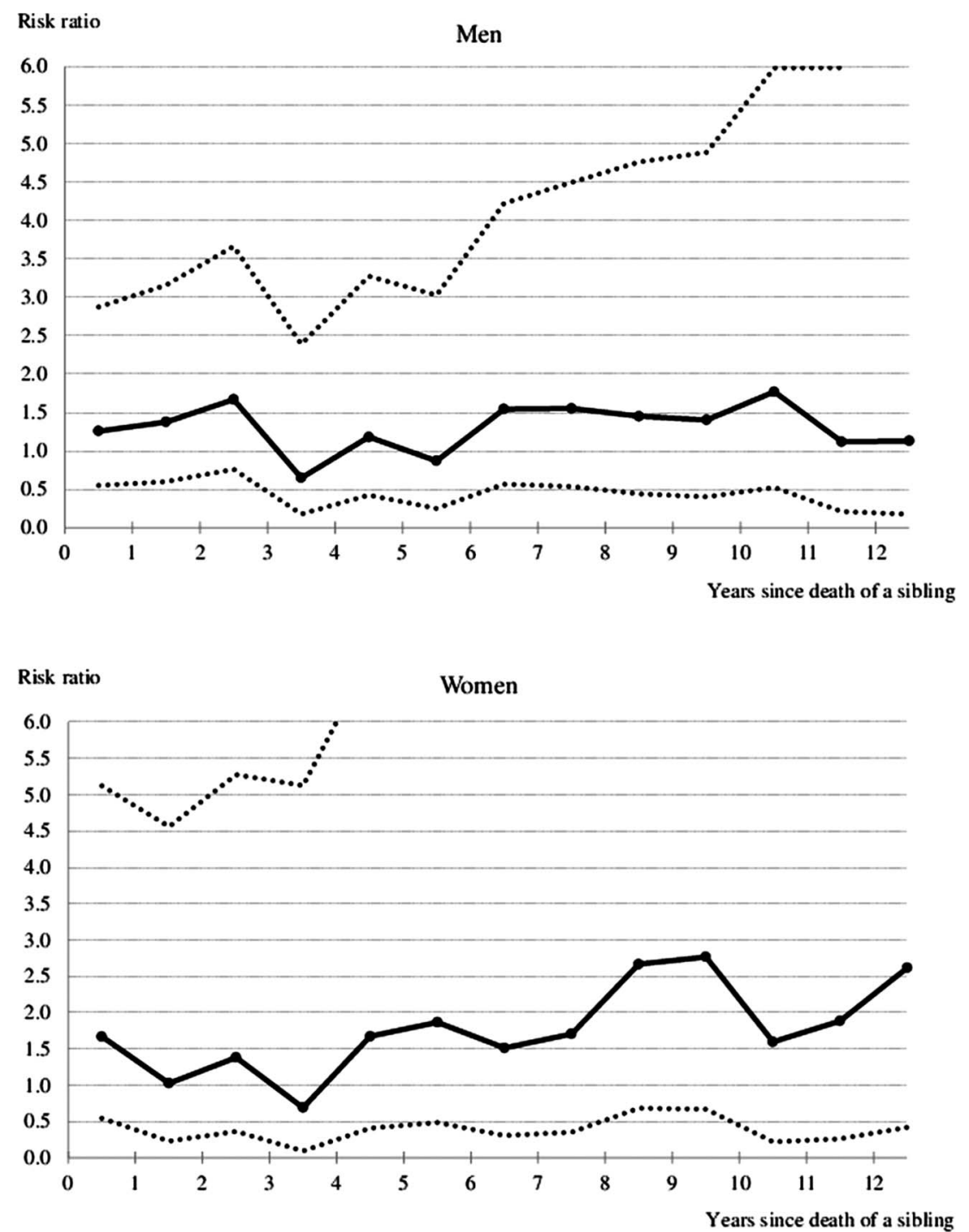

risk after a sibling's death remained fairly stable, whereas in women there was a slight tendency towards an increase in the suicide risk according to time since bereavement. These findings should be interpreted with utmost caution as the CIs are very wide due to the relatively small number of deaths.

\section{DISCUSSION}

According to the $\mathrm{WHO}$, almost one million people die from suicide each year, and suicide is among the three leading causes of death for people aged less than 25 years. $^{27}$ To prevent and identify risk factors for suicide is therefore of utmost importance.

This large-scale follow-up study based on the Swedish population register examined suicide following the death of a sibling. We found an increased suicide risk among women and men who had experienced the suicide of a sibling, whereas associations with other main causes of death were generally much lower and statistically not significant. Women appeared somewhat more vulnerable than men with respect to how their suicide risk relates to a sibling's death and a sibling's suicide, in particular. We found no clear support for a specific time pattern according to time since a sibling's death. In men, the excess suicide risk seemed fairly stable over time, whereas among women there were some indications of an increase according to time since the sibling's death.

Although interpersonal loss and disruption of attachments from any cause could elevate the risk for suicide, ${ }^{13-15}$ the findings of this study suggest that deaths from suicide in particular are most detrimental. Relatives exposed to suicide, accidents or violent deaths-which are often sudden and unanticipated-might be at greater risk of developing complicated grief and post-traumatic stress symptoms which could explain their higher suicide risk. ${ }^{132122}$ In line with this, deaths from cancer and cardiovascular disease showed no significant associations with suicide risk of bereaved siblings. These chronic diseases tend to run a longer course and therefore allow relatives to adjust and adapt to the circumstances.

It has been argued that the pain of dealing with the loss of a loved one by suicide is especially severe when compared with other types of external deaths such as 
accidents and homicides. This might explain why we found particularly strong associations between sibling's suicide and suicide among bereaved siblings. There are qualitative aspects of the mourning process that are intensified and frequently more problematic for survivors of suicide than for other types of bereavement. ${ }^{13} 28$ Survivors of suicide seem to struggle more with questions of meaning around the death. ${ }^{29}{ }^{30}$ Survivors often show higher levels of guilt, blame and responsibility for the death than other mourners. ${ }^{31}{ }^{32}$ Studies also find that survivors experience feelings of rejection and abandonment by the loved one, along with anger towards the deceased. ${ }^{33}$ However, death by suicide is also stigmatising to surviving family members and initiates a chain reaction of negative consequences ${ }^{31}$ that may lead to an unsatisfactory resolution of bereavement. It has been found that suicide survivors received less emotional support than natural death survivors for their feelings of depression and grief and confided less in members of their social networks. ${ }^{34}$

Women's risk of suicide was found to be somewhat higher after the death of a sibling from suicide when compared with men. This finding might reflect the fact that women place more emphasis on social relationships than men do, particularly when it comes to parents and the family. ${ }^{35}$ The loss of a sibling could hence have stronger emotional consequences for women which, in turn, could account for a higher risk of suicide. The longer-term association found among women may also suggest that longer-term mechanisms such as an extended and complicated grief process and/or severe depression underlie the association. In a previous study, we found that women's health is more influenced by bereavement following sibling loss than men's health. ${ }^{5}$

Siblings also share many environmental exposures during childhood and adolescence. Suicide has been associated with family risk factors for suicide such as disorganisation and break-up, parental loss, substance abuse, intrafamily violence and sexual abuse. ${ }^{13}$ Deaths from external causes other than suicide, such as accidents and homicides, could, however, also reflect risktaking behaviours due to depression, psychiatric disorders and shared environmental exposures. Consequently, genetic confounding might account for a higher risk of suicide following sibling deaths from external causes. Given the strong genetic similarities between siblings, there could be a higher risk of confounding when compared with research on other types of bereavement. Considering that the association between bereavement by suicide and suicide in bereaved siblings is the strongest observed in this study, it could also reflect the 'copycat phenomenon' that is, suicides in bereaved siblings could be caused by imitation of suicidal behaviour. ${ }^{36}$ Nevertheless, our methodological strategy accounted for intrafamily correlation in death risks which is a great strength of the study. This is important, considering that genetic factors and shared early-life experiences can predispose people towards the development of psychiatric disorders that are associated with suicide, particularly depression and bipolar disorders. ${ }^{14}$

\section{LIMITATIONS}

Despite the obvious strengths of this study, such as the use of TPR data, a large sample size, longitudinal follow-up, reliable information on deaths from suicide and other included variables, some limitations should be noted. More detailed individual information is required to uncover the actual causal mechanisms that link sibling suicide and mortality. Such information could also minimise the possibility of omitted variable bias. Ideally, one would like to have access to biological and genetic data, detailed information on diseases from medical records, more information on shared childhood social environment and family characteristics and detailed data on personal and relational characteristics, which are unfortunately not included in the registers. In particular, the inability of registry-based studies to attend for family-level risk factors such as the quality of sibling relationship is a limitation of the study. The quality of the relationship with the deceased sibling could decide the severity of bereavement, and thereby contribute to suicide risk among the remaining siblings. The study also involves the lack of information on surviving sibling's medical and psychiatric status, both of which convey important information about suicide risk, and temporal relationship of medical illnesses and mental health disorder episodes to both bereavement and suicide. On the other hand, our results likely underestimate the true bereavement effect following sibling death from suicide, since we could study only completed suicide, and not suicide attempts which are much more common. ${ }^{37}$ Examining the attempted suicide is likely to provide more precision and even greater statistical power.

Our findings suggest that the healthcare system should consider collateral health effects when dealing with people exposed to sibling deaths from external causes. Most discussion has focused on bereavement after the death of a spouse or a parent, while siblings have tended to be the 'forgotten grievers'. Our findings illustrate that a sibling's death due to suicide can increase the risk of suicide among bereaved siblings. Considering that their loss and pain are often insufficiently acknowledged by the parents and the informal social support system, ${ }^{11}{ }^{12}$ it is important that physicians and healthcare professionals acknowledge bereaved siblings from the causes of death such as suicide, accidents and violent deaths. Accordingly, targeted support towards bereaved siblings who experienced sibling deaths from suicide may be beneficial and reduce their risk of suicide. Our findings also suggest the need for further research to determine whether long-term follow-up and support provided by healthcare workers to surviving siblings can reduce suicide risk.

In summary, our study provided evidence for suicide associated with the death of a sibling at adult age and illustrated that bereaved siblings' risk of suicide is 
primarily influenced by deaths from suicide. The mechanisms linking the death of a sibling and completed suicide among the bereaved person need to be further investigated.

Contributors MR and JS planned the study and the research design. They also analysed the data, interpreted the results and wrote the draft version of the paper. IK contributed to writing, interpretation of data and critical reviews. All authors corrected and approved the final version of the manuscript.

Funding MR was supported by the Swedish Council for Working Life and Social Research (grant number 2009-0547) and the Swedish Research Council (grant number 421-2011-1649). The funding source(s) had no involvement in the study design, the collection of data, analysis, interpretation of data, writing of the report or in the decision to submit the paper for publication.

Competing interests None.

Ethics approval The Regional Ethical Review Board of Karolinska Institutet in 2002-11-11 (decision no. 02-481) and the Central Ethical Review Board 2012-09-13 (application no. 2012/1260-31).

Provenance and peer review Not commissioned; externally peer reviewed.

Data sharing statement No additional data are available.

\section{REFERENCES}

1. Li J, Precht DH, Mortensen PB, et al. Mortality in parents after death of a child in Denmark: a nationwide follow-up 445 study. Lancet 2003;361:363-7.

2. Martikainen $\mathrm{P}$, Valkonen T. Mortality after the death of a spouse: rates and causes of death in a large Finnish cohort. Am J Public Health 1996;86:1087-93.

3. Hendrickson KC. Morbidity, mortality, parental grief: a 450 review of the literature on the relationship between the death of a child the subsequent health of parents. Palliat Support Care 2009;7:109-19.

4. Rostila M, Saarela JM. Time does not heal all wounds: mortality following the death of a parent. J Marriage Fam 2011;73:236-49.

5. Rostila M, Saarela J, Kawachi I. The forgotten griever: a nationwide follow-up study of mortality subsequent to the death of a sibling. Am J Epidemiol 2012;176:338-46.

6. Bunch J. Recent bereavement in relation to suicide. J Psychosom Res 1972;16:361-6.

7. Kaprio J, Koskenvuo M, Rita H. Mortality after bereavement: a prospective study of 95647 widowed persons. Am J Public Health 1987;77:283-7.

8. Erlangsen A, Jeune B, Bille-Brahe $U$, et al. Loss of partner and suicide risks among oldest old: a population-based register study. Age Ageing 2004;33:378-83.

9. Qin P, Mortensen PB. The impact of parental status on the risk of completed suicide. Arch Gen Psychiatry 2003;60:797-802.

10. Li J, Precht DH, Mortensen PB, et al. Mortality in parents after death of a child in Denmark: a nationwide follow-up study. Lancet 2003;361:363-7.

11. Robinson L, Mahon MM. Sibling bereavement: a concept analysis. Death Stud 1997;21:477-99.

12. Segal NL, Wilson SM, Bouchard TJ, et al. Comparative grief experiences of bereaved twins and other bereaved relatives. Person Individ Diff 1995;18:511-24.

13. Jordan JR. Is suicide bereavement different? A reassessment of the literature. Suicide Life Threat Behav 2001;31:91-102.
14. Moscicki EK. Epidemiology of suicidal behavior. Suicide Life Threat Behav 1995;25:22-35.

15. Heikkinen M, Aro H, Lönnqvist J. Life events and social support in suicide. Suicide Life Threat Behav 1993;23:343-58.

16. Brown GW. Loss and depressive disorders. In: Dohrenwend B, ed Adversity, stress, and psychopathology. New York: Oxford University Press, 1998:358-70.

17. Jacobs S. Traumatic grief: diagnosis, treatment, and prevention. Philadelphia: Taylor \& Francis, 1999.

18. Amick-McMullan A, Kilpatrick DG, Resnick HS. Homicide as a risk factor for PTSD among surviving family members. Behav Modif 1991;15:545-59.

19. Murphy SA, Johnson LC, Wu L, et al. Bereaved parents' outcomes 4-60 months after their children's deaths by accidents, suicide or homicide: a comparative study demonstrating differences. Death Stud 2003;27:39-61.

20. Brent D, Melhem N, Donohoe MB, et al. The incidence and course of depression in bereaved youth 21 months after the loss of a parent to suicide, accident, or sudden natural death. Am J Psychiatry 2009;166:786-94.

21. Dyregrov K, Nordanger D, Dyregrov A. Predictors of psychosocial distress after suicide, SIDS and accidents. Death Stud 2003;27:143-65.

22. Nakajima $S$, Ito $M$, Shirai $A$, et al. Complicated grief in those bereaved by violent death: the effects of post-traumatic stress disorder on complicated grief. Dialogues Clin Neurosci 2012;14:210-14.

23. Merlevede E, Spooren D, Henderick H, et al. Perceptions, needs and mourning reactions of bereaved relatives confronted with a sudden unexpected death. Resuscitation 2004;61:341-8.

24. Parkes CM. Bereavement in adult life. BMJ 1998;316:856-9.

25. Bowling A. Mortality after bereavement: a review of the literature on survival periods and factors affecting survival. Soc Sci Med 1987;24:117-24.

26. Vaida F, Xu R. Proportional hazards model with random effects. Stat Med 2000;19:3309-24.

27. World Health Organization (WHO). http://www.who.int/ mental_health/prevention/suicide/suicideprevent/en/ (accessed 15 Oct 2012).

28. Calhoun LG, Selby JW, Abernathy CB. The rules of bereavement: are suicidal deaths different? J Community Psych 1986;14:213-18.

29. Van der Wal J. The aftermath of suicide: a review of empirical evidence. Omega 1989-1990;20:149-71.

30. Grad O, Zavasnik A. Similarities and differences in the process of bereavement after suicide and after traffic fatalities in Slovenia. Omega 1996;33:243-51.

31. Demi AS. Social adjustment of widows after a sudden death: suicide and non-suicide survivors compared. Death Educ 1984;8:91-111.

32. Silverman E, Range L, Overholser J. Bereavement from suicide as compared to other forms of bereavement. Omega 1995;30:41-51.

33. Barrett TW, Scott TB. Suicide bereavement and recovery patterns compared with non-suicide bereavement patterns. Suicide Life Threat Behav 1990;20:1-15.

34. Farberow N, Gallagher-Thompson D, Gilewski M, et al. The role of social support in the bereavement process of surviving spouses of suicide and natural deaths. Suicide Life Threat Behav 1992;22:107-24.

35. Umberson D, Meichu C, House J, et al. The effect of social relationships on psychological well-being: are men and women really so different? Am Sociol Rev 1996;61:837-57.

36. Mesoudi A. The cultural dynamics of copycat suicide. PLoS One 2009;4:e7252

37. Isometsä ET, Lönnqvist JK. Suicide attempts preceding completed suicide. Br J Psychiatry 1998;173:531-5. 